Algebraic $8 \mathcal{G}$ Geometric $\mathcal{T}_{\text {opology }}$

Volume 4 (2004) 219-241

Published: 13 April 2004

ATG

\title{
Adem relations in the Dyer-Lashof algebra and modular invariants
}

\author{
Nondas E. KeChagias
}

\begin{abstract}
This work deals with Adem relations in the Dyer-Lashof algebra from a modular invariant point of view. The main result is to provide an algorithm which has two effects: Firstly, to calculate the hom-dual of an element in the Dyer-Lashof algebra; and secondly, to find the image of a non-admissible element after applying Adem relations. The advantage of our method is that one has to deal with polynomials instead of homology operations. A moderate explanation of the complexity of Adem relations is given.
\end{abstract}

AMS Classification 55S10, 13F20; 55P10

Keywords Adem relations, Dyer-Lashof algebra, Dickson algebra, Borel invariants

\section{Introduction}

The relationship between the (canonical sub-co-algebras) Dyer-Lashof algebra, $R[k]$ and the Dickson invariants $D[k]$ is well-known, see May's paper in [3], relevant parts of which will be quoted here. We provide an algorithm for calculating Adem relations in the Dyer-Lashof algebra using modular co-invariants. Much of our work involves the calculation of the hom-duals of elements of $R$ in terms of the generators of the polynomial algebra $D[k]$. The results described here will be applied to give an invariant theoretic description of the $\bmod -p$ cohomology of a finite loop space in [6].

We note that the idea for our algorithm was inspired by May's theorem 3.7, page 29 , in [3]. The key ingredient for relating homology operations and polynomial invariants is the relation between the map which imposes Adem relations and the decomposition map between certain rings of invariants. This relation was studied by Mui for $p=2$ in [8], and we extend it here for any prime. Namely: 
Theorem 4.15 Let $\rho: T[n] \rightarrow R[n]$ be the map which imposes Adem relations. Let $\hat{\imath}: S(E(n))^{G L_{n}} \otimes D[n] \hookrightarrow S(E(n))^{B_{n}} \otimes B[n]$ be the natural inclusion. Then $\rho^{*} \equiv \hat{\imath}$, i.e. for any $e_{I} \in T[n]$ and $d^{m} M^{\varepsilon} \in S(E(n))^{G L_{n}} \otimes D[n]$,

$$
\left\langle d^{m} M^{\varepsilon}, \rho\left(e_{I}\right)\right\rangle=\left\langle\hat{\imath}\left(d^{m} M^{\varepsilon}\right), e_{I}\right\rangle .
$$

Campbell, Peterson and Selick studied self maps $f$ of $\Omega_{0}^{m+1} S^{m+1}$ and proved that if $f$ induces an isomorphism on $H_{2 p-3}\left(\Omega_{0}^{m+1} S^{m+1}, \mathbb{Z} / p \mathbb{Z}\right)$, then $f_{(p)}$ is a homotopy equivalence for $p$ odd and $m$ even [2] A key ingredient for their proof was the calculation of

$$
\operatorname{AnnPH} H^{*}\left(\Omega_{0}^{m+1} S^{m+1}, \mathbb{Z} / p \mathbb{Z}\right)
$$

They gave a convenient method for calculating the hom-dual of elements of $H_{*}\left(\Omega_{0}^{m+1} S^{m+1}, \mathbb{Z} / p \mathbb{Z}\right)$ which do not involve Bockstein operations. Our algorithm computes the hom-duals of elements of $R[n]$ in terms of the generators of the polynomial algebra $D[n]$. Please see Theorem 4.16 .

A direct application of the last two theorems is the computation of Adem relations. The main difference between the classical and our approach is that we consider Adem relations "globally" instead of consecutive elements and it requires fewer calculations. This algorithm is described in Proposition 4.20,

The paper is purely algebraic and its applications are deferred to 6]. There are three sections in this paper beyond this introduction, sections 2, 3 and 4 . Section 2 recalls well known facts about the Dyer-Lashof algebra from May's article, cited above. In section 3, the Dickson algebra and its relation with the ring of invariants of the Borel subgroup is examined. That relation is studied using a certain family of matrices which suitably summarizes the expressions for Dickson invariants in terms of the invariants of the Borel subgroup. In the view of the author, the complexity of Adem relations is reflected in the different ways in which the same monomial in the generators of the Borel subgroup can show up as a term in a Dickson invariant. The ways in which this can happen can be understood using these matrices. For $p$ odd, the dual of the Dyer-Lashof algebra is a subalgebra of the full ring of invariants. This subalgebra is also discussed in full details. In the last section a great amount of work is devoted to the proof of the analog of Mui's result mentioned above. Then our algorithms more or less naturally follows.

This paper has been written for odd primes with minor modifications needed when $p=2$ provided in statements in square brackets following the odd primary statements. 
For the sake of accessibility we shorten proofs. A detailed version of this work including many examples can be found at:

http://msp.warwick.ac.uk/agt/2004/04/x013/full.pdf

This work is dedicated to the memory of Professor F.P. Peterson.

We thank Eddy Campbell very much for his great effort regarding the presentation and organization of the present work and the referee for his encourangment and valuable suggestions regarding the accessibility of our algorithm to the interested reader. Last but not least, we thank the editor very much.

\section{The Dyer-Lashof algebra}

Let us briefly recall the construction of the Dyer-Lashof algebra. Let $F$ be the free graded associative algebra on $\left\{f^{i}, i \geq 0\right\}$ and $\left\{\beta f^{i}, i>0\right\}$ over $K:=\mathbb{Z} / p \mathbb{Z}$ with $\left|f^{i}\right|=2(p-1) i,\left[\left|f^{i}\right|=i\right]$ and $\left|\beta f^{i}\right|=2 i(p-1)-1 . \quad F$ becomes a co-algebra equipped with coproduct $\psi: F \longrightarrow F \otimes F$ given by

$$
\psi f^{i}=\sum f^{i-j} \otimes f^{j} \text { and } \psi \beta f^{i}=\sum \beta f^{i-j} \otimes f^{j}+\sum f^{i-j} \otimes \beta f^{j} .
$$

Elements of $F$ are of the form

$$
f^{I, \varepsilon}=\beta^{\epsilon_{1}} f^{i_{1}} \ldots \beta^{\epsilon_{n}} f^{i_{n}}
$$

where $(I, \varepsilon)=\left(\left(i_{1}, \ldots, i_{n}\right),\left(\epsilon_{1}, \ldots, \epsilon_{n}\right)\right)$ with $\epsilon_{j}=0$ or 1 and $i_{j}$ a non-negative integer for $j=1, \ldots, n,\left|f^{I, \varepsilon}\right|=2(p-1)\left(\sum_{t=1}^{n} i_{t}\right)-\left(\sum_{t=1}^{n} e_{t}\right)\left[\left|f^{I, \varepsilon}\right|=\left(\sum_{t=1}^{n} i_{t}\right)\right]$. Let $l(I, \varepsilon)=n$ denote the length of $I, \varepsilon$ or $f^{I, \varepsilon}$ and let the excess of $(I, \varepsilon)$ or $f^{I, \varepsilon}$ be denoted $\operatorname{exc}\left(f^{I, \varepsilon}\right)=i_{1}-\epsilon_{1}-\left|f^{I_{2}}\right|$, where $\left(I_{t}, \varepsilon_{t}\right)=\left(\left(i_{t}, \ldots, i_{n}\right),\left(\epsilon_{t}, \ldots, \epsilon_{n}\right)\right)$.

$$
\operatorname{exc}\left(f^{I, \varepsilon}\right)=i_{1}-\epsilon_{1}-2(p-1) \sum_{2}^{n} i_{t},\left[\operatorname{exc}\left(f^{I}\right)=i_{1}-\sum_{2}^{n} i_{t}\right]
$$

The excess is defined $\infty$, if $I=\emptyset$ and we omit the sequence $\left(\epsilon_{1}, \ldots, \epsilon_{n}\right)$, if all $e_{i}=0$. We refer to elements $f^{I}$ as having non-negative excess, if $\operatorname{exc}\left(f^{I_{t}}\right)$ is non-negative for all $t$.

It is sometimes convenient to use lower notation for elements of $F$ and its quotients. We define $f^{i} x=f_{\frac{1}{2}(2 i-|x|)} x\left[f^{i} x=f_{i-|x|} x\right]$. Let $I=\left(i_{1}, \ldots, i_{n}\right)$ and $\varepsilon=\left(\epsilon_{1}, \ldots, \epsilon_{n}\right)$, then the degree of $Q_{I, \varepsilon}$ is

$$
\left|f_{I, \varepsilon}\right|=2(p-1)\left(\sum_{t=1}^{n} i_{t} p^{t-1}\right)-\left(\sum_{t=1}^{n} e_{t} p^{t-1}\right),\left[\left|f_{I, \varepsilon}\right|=\left(\sum_{t=1}^{n} i_{t} 2^{t-1}\right)\right] .
$$


In lower notation we see immediately that $f_{I, \varepsilon}$ has non-negative excess if and only if $(I, \varepsilon)$ is a sequence of non-negative integers: $\operatorname{exc}(I, \varepsilon)=2 i_{1}-e_{1}$.

Given sequences $I$ and $I^{\prime}$ we call the direct sum of $I$ and $I^{\prime}$ the sequence $I \oplus I^{\prime}=\left(i_{1}, \ldots, i_{n}, i_{1}^{\prime}, \ldots, i_{m}^{\prime}\right)$. Using a sequence $I$ we use the above idea for the appropriate decomposition. Let $0_{k}$ denote the zero sequence of length $k$.

Remark Let $\left\langle\mathbb{N}, \frac{1}{2}\right\rangle$ be the monoid generated by $\mathbb{N}$ and $\frac{1}{2}$ in the rationals. Let $\left\langle\mathbb{N}, \frac{1}{2}\right\rangle^{n}$ be the monoid which is the $n$-th Cartesian product of $\left\langle\mathbb{N}, \frac{1}{2}\right\rangle$. Then $(I, \varepsilon) \in\left\langle\mathbb{N}, \frac{1}{2}\right\rangle^{n} \times(Z / 2 Z)^{n} \cdot\left[I \in \mathbb{N}^{n}\right]$

$F$ admits a Hopf algebra structure with unit $\eta: K \longrightarrow F$ and augmentation $\epsilon: F \longrightarrow K$ given by:

$$
\epsilon\left(f^{i}\right)= \begin{cases}1, & \text { if } i=0 \\ 0, & \text { otherwise }\end{cases}
$$

Definition 2.1 There is a natural order on the elements $f_{(I, \varepsilon)}$ defined as follows: for $(I, \varepsilon)$ and $\left(I^{\prime}, \varepsilon^{\prime}\right)$ we say that $(I, \varepsilon)<\left(I^{\prime}, \varepsilon^{\prime}\right)$ if $\operatorname{exc}\left(I_{l}, \varepsilon_{l}\right)=\operatorname{exc}\left(I_{l}^{\prime}, \varepsilon_{l}^{\prime}\right)$ for $1 \leq l \leq t$ and $\operatorname{exc}\left(I_{t}, \varepsilon_{t}\right)<\operatorname{exc}\left(I_{t}^{\prime}, \varepsilon_{t}^{\prime}\right)$ for some $1 \leq t \leq n$.

We define $T=F / \mathcal{I}_{\text {exc }}$, where $\mathcal{I}_{\text {exc }}$ is the two sided ideal generated by elements of negative excess. $T$ inherits the structure of a Hopf algebra and if we let $T[n]$ denote the set of all elements of $T$ with length $n$, then $T[n]$ is a co-algebra of finite type. We denote the image of $f_{I, \varepsilon}$ by $e_{I, \varepsilon}$. Degree, excess and ordering for upper or lower notation described above passes to $T$ and $T[n]$.

The Adem relations are given by:

$$
e_{r} e_{s}=\sum_{i}(-1)^{\mathbf{r}-i}\left(\begin{array}{c}
(p-1)(i-s)-1 \\
r-i-1
\end{array}\right) e_{r+p s-p i} e_{i}, \text { if } r>s
$$

and if $p>2$ and $r \geq s$,

$$
\begin{gathered}
e_{r} \beta e_{s}=\sum_{i}(-1)^{r+i+1 / 2}\left(\begin{array}{c}
(p-1)(i-s) \\
r-1 / 2-i
\end{array}\right) \beta e_{r+p s-p i-1 / 2} e_{i}+ \\
\sum_{i}(-1)^{r+i-1 / 2}\left(\begin{array}{c}
(p-1)(i-s)-1 \\
r-1 / 2-i
\end{array}\right) e_{r+p s-p i} \beta e_{i} .
\end{gathered}
$$

Let $\mathcal{I}_{\text {Adem }}$ be the two sided ideal of $T$ generated by the Adem relations. We denote $R$ the quotient $T / \mathcal{I}_{\text {Adem }}$ and this quotient algebra is called the DyerLashof algebra. $R$ is a Hopf algebra and $R[n]$ is again a co-algebra of finite 
type. We will denote the obvious epimorphism above which imposes Adem relations by

$$
\rho: T \rightarrow R \text { with } \rho\left(e_{I}\right)=\sum a_{I, J} Q_{J}
$$

If $(I, \varepsilon)$ is admissible then $Q_{I, \varepsilon}$ is the image of $e_{I, \varepsilon}$.

The following lemma will be applied in section 4 .

Lemma 2.2 a) $\rho\left(e_{p^{k}} e_{0}\right)=Q_{0} Q_{p^{k-1}} ; \rho\left(e_{1} e_{0}\right)=0$.

b) $\rho\left(e_{p^{k}+1 / 2} e_{1 / 2}\right)=Q_{1 / 2} Q_{p^{k-1}+1 / 2} ; \rho\left(e_{3 / 2} e_{1 / 2}\right)=0$.

c) $\rho\left(e_{p^{k}+1} e_{1}\right)=Q_{1} Q_{p^{k-1}+1} ; \rho\left(e_{2} e_{1}\right)=0$.

d) $\rho\left(e_{p^{k}} e_{1}\right)=Q_{0} Q_{p^{k-1}+1} ; \rho\left(e_{p} e_{1}\right)=2 Q_{0} Q_{2}$.

e) $\rho\left(e_{p^{k}} \beta e_{1 / 2}\right)=Q_{0} \beta Q_{p^{k-1}+1 / 2} ; \rho\left(e_{1} \beta e_{1 / 2}\right)=\beta Q_{1 / 2} Q_{1 / 2}$.

f) $\rho\left(e_{p^{k}+1 / 2} e_{1 / 2}\right)=0 ; \rho\left(e_{3 / 2} \beta e_{1}\right)=\beta Q_{1} Q_{1}$.

g) $\rho\left(e_{p^{k}+1} \beta e_{1 / 2}\right)=\beta Q_{1 / 2} Q_{p^{k-1}+1 / 2} ; \rho\left(e_{2} \beta e_{1 / 2}\right)=0$.

The passage from lower to upper notation between elements of $R$ is given as follows. Let $J x \varepsilon$ and $I x \varepsilon$ be lower and upper sequences as defined above. Then,

$$
\beta^{\epsilon_{1}} Q_{j_{1}} \ldots \beta^{\epsilon_{n}} Q_{j_{n}} \equiv \beta^{\epsilon_{1}} Q^{i_{1}} \ldots \beta^{\epsilon_{n}} Q^{i_{n}}
$$

up to a unit in $\mathbb{Z} / p \mathbb{Z}$, where $i_{n}=j_{n}$, and

$$
i_{n-t}=\frac{1}{2}\left(2 j_{n-t}+\left|I_{n-t+1} x \varepsilon_{n-t+1}\right|\right), j_{n-t}=\frac{1}{2}\left(2 i_{n-t}-\left|J_{n-t+1} x \varepsilon_{n-t+1}\right|\right)
$$

Definition 2.3 We say that an element $Q_{I, \varepsilon}$ is admissible, if $0 \leq 2 i_{t}-2 i_{t-1}+$ $e_{t-1}$ for $2 \leq t \leq n-1$.

The ordering described above passes to $R$ and $R[n]$.

Since $R[n]$ and $T[n]$ are of finite type, they are isomorphic to their duals as vector spaces and these duals become algebras. We shall describe these duals giving an invariant theoretic description, namely: they are isomorphic to subalgebras of rings of invariants over the appropriate subgroup of $G L(n, K)$ in section 4 . 


\section{The Dickson algebra and a special family of ma- trices}

The Dickson algebra is a universal object in modular invariants of finite groups. Applications involve computations of Dickson invariants of different height. We provide formulas of this nature which will be applied in the proof of Theorem 4.16 Being very technical, those formulas can be studied easier using matrices.

Let $V^{k}$ denote a $K$-dimensional vector space generated by $\left\{e_{1}, \ldots, e_{k}\right\}$ for $1 \leq k \leq n$. Let the dual basis of $V^{n}$ be $\left\{x_{1}, \ldots, x_{n}\right\}$ and the contragradient representation of $W_{\Sigma_{p^{n}}}\left(V^{n}\right) \longrightarrow \operatorname{Aut}\left(V^{n}\right) \equiv G L_{n}$ induces an action of $G L_{n}$ on the graded algebra $E\left(x_{1}, \ldots, x_{n}\right) \otimes P\left[y_{1}, \ldots, y_{n}\right],\left[P\left[y_{1}, \ldots, y_{n}\right]\right]$, where $\beta x_{i}=y_{i}$. Let $E(n)=E\left(x_{1}, \ldots, x_{n}\right)$ and $S[n]=K\left[y_{1}, \cdots, y_{n}\right]$. The degree is given by $\left|x_{i}\right|=1$ and $\left|y_{i}\right|=2$ (if $p=2$, then $\left|y_{i}\right|=1$ ).

The following theorems are well known:

Theorem 3.1 4] $S[n]^{G L_{n}}:=D[n]=K\left[d_{n, 0}, \cdots, d_{n, n-1}\right]$, the Dickson algebra, is a polynomial algebra and their degrees are $\left|d_{n, i}\right|=2\left(p^{n}-p^{i}\right),\left[2^{n}-2^{i}\right]$.

Theorem 3.2 [7] $S[n]:=B[n]=K\left[h_{1}, \cdots, h_{n}\right]$ is a polynomial algebra and their degrees are $\left|h_{i}\right|=2 p^{i-1}(p-1),\left[2^{i-1}\right]$.

Although relations between generators of the last two algebras can be easily described, it is not the case between invariants of parabolic subgroups of the general linear group.

Let $f_{k-1}(x)=\prod_{u \in V^{k-1}}(x-u)$, then $f_{k-1}(x)=\sum_{i=0}^{k-1}(-1)^{n-i} x^{p^{i}} d_{k-1, i}$ and $h_{k}=$ $\prod_{u \in V^{k-1}}\left(y_{k}-u\right)$. Moreover, (see [5]),

$$
d_{n, n-i}=\sum_{1 \leq j_{1}<\cdots<j_{i} \leq n} \prod_{s=1}^{i}\left(h_{j_{s}}\right)^{p^{n-i+s-j_{s}}}
$$

Let $m=\left(m_{0}, \ldots, m_{n-1}\right)$ and $k=\left(k_{1}, \ldots, k_{n}\right)$ be sequences of non-negative integers. Let $d^{m}$ denote an element of $D[n]$ given by $\prod_{t=0}^{n-1} d_{n, t}^{m_{t}}$ and $h^{k}$ denote an element of $B[n]$ given by $\prod_{t=1}^{n} h_{t}^{k_{t}}$. Let $I_{(t)}$ denote the $t$-th element of the sequence $I=\left(i_{l_{1}}, \ldots, i_{l_{n}}\right)$ from the left: i.e. $I_{(t)}:=i_{l_{t}}$.

For any non-negative matrix $C$ with integral entries and $\mathbf{1}=(1, \ldots, 1)$, the matrix product $\mathbf{1} \cdot C$ is a sequence of non-negative integers, then $h^{1 \cdot C}$ stands 
for $\prod_{t=1}^{n} h_{t}^{(\mathbf{1} \cdot C)_{(t)}}$ Let $C\left(d_{n, j}\right)=\left\{h^{I} \in B[n]\right.$ and $h^{I}$ is a non-trivial summand in $\left.d_{n, j}\right\}$, then $C\left(d_{n, i}\right) \cap C\left(d_{n, j}\right)=\emptyset$ for $j \neq i$.

Remark 1) Before we start considering sets of matrices, we would like to stress the point that the zero matrix is excluded from our sets, unless otherwise stated.

2) Until the end of this section, we number matrices beginning with $(0,0)$ in the upper left corner. In this case $h^{\mathbf{1} \cdot C}$ stands for $\prod_{t=1}^{n} h_{t}^{(\mathbf{1} \cdot C)_{(t-1)}}$.

Let $0 \leq j \leq n-1$. Here $j$ corresponds to the value $n-i$ in formula 1 .

Definition 3.3 For each matrix $A=\left(a_{i t}\right)$ such that $a_{i t}$ is a non-negative integer, $\sum_{t=0}^{n-1} a_{j t}=n-j$ and $\sum_{t=0}^{n-1} a_{i t}=0$ for $i \neq j$, we define an $n \times n$ matrix $C(A)=\left(b_{i j}\right)=\left(b_{(0)}, \cdots, b_{(n-1)}\right)$ such that $b_{i t}=a_{i t} p^{i-1-t+a_{i 0}+\cdots+a_{i t}}$. Let us call this collection $A_{n, j}$.

For $C \in A_{n, j}, \mathbf{1} \cdot C$ is the $j$-th row of $C$ which is the only non-zero row of that matrix.

Let us also note that there is an obvious bijection between $A_{n, j}$ and $C\left(d_{n, j}\right)$.

Lemma $3.4 d_{n, j}=\sum_{C \in A_{n, j}} h^{1 \cdot C}$.

Definition 3.5 Let $m=\left(m_{0}, \cdots, m_{n-1}\right)$ be a sequence of zeros or powers of $p$. Let $A_{n, j}^{m}=\left\{m \cdot C_{j}=\left(m_{0} b_{(0)}, \cdots, m_{n-1} b_{(n-1)}\right) \mid C_{j}=\left(b_{(0)}, \cdots, b_{(n-1)}\right) \in A_{n, j}\right\}$ and $A_{n}^{m}=\left\{\sum_{j=0}^{n-1} m \cdot C_{j} \mid C_{j} \in A_{n, j}\right\}$.

Note that different elements of $A_{n}^{m}$ may provide the same element of $B[n]$ and this is the reason why Adem relations are complicated as we shall examine more in Proposition 3.10. We shall also note that the motivation of this section was exactly to demonstrate this difficulty using an elementary method.

The following lemma is easily deduced from formulae 1.

Lemma 3.6 Let $m=\left(m_{0}, \cdots, m_{n-1}\right)$ such that $m_{i}=0$ or $p^{k_{i}}$, then

$$
d^{m}=\prod_{0}^{n-1} d_{n, i}^{m_{i}}=\sum_{C \in A_{n}^{m}} \prod_{t=1}^{n}\left(h_{t}\right)^{(C(\mathbf{1}))_{t-1}} .
$$


Coefficients might appear in the last summation. Hence one needs to partition the set $A_{n}^{m}$ as the following lemma suggests.

Lemma 3.7 Let $m=\left(m_{0}, \cdots, m_{n-1}\right)$ be a sequence of zeros or powers of $p$. Let $A=\left(a_{i t}\right)$ and $A^{\prime}=\left(a_{i t}^{\prime}\right)$ such that $a_{i t}, a_{i t}^{\prime} \in \mathbb{N}, \sum_{t=0}^{n-1} a_{j t}=\sum_{t=0}^{n-1} a_{j t}^{\prime}=n-j$ if $m_{i} \neq 0$, otherwise the last sums are zero. Suppose that $\mathbf{1} \cdot A=\mathbf{1} \cdot A^{\prime}$ and let $\left\{i_{1}, \ldots, i_{q}\right\}$ denote their different columns. Consider only their different rows and for each column $i_{r}$ partition them according to where 1 's appear: $\left\{j_{1}, \ldots, j_{s}\right\}$ and $\left\{j_{1}^{\prime}, \ldots, j_{s}^{\prime}\right\}$. If for each $j_{t}$ there exists a $j_{\ell}^{\prime}$ such that the number of zeros next to $a_{i_{r}, j_{t}}$ and $a_{i_{r}, j_{t}^{\prime}}^{\prime}$ are equal and this is true for all $i_{r}$, then $\mathbf{1} \cdot C(A)=\mathbf{1} \cdot C\left(A^{\prime}\right)$.

Proof We use the definition of $C(A)$ in 3.3 .

On $1 x n$ or $n x 1$ matrices we give the left or upper lexicographical ordering respectively.

Definition 3.8 Let $m$ be a non-negative integer, we denote by $\left|A_{n, j}\right|(m)$ the set of partitions of $m$ in $\left|A_{n, j}\right|$ terms. A typical element of $\left|A_{n, j}\right|(m)$ is of the form $\pi=\left(\pi_{1}, \ldots, \pi_{\left|A_{n, j}\right|}\right)$.

For $\pi=\left(\pi_{1}, \ldots, \pi_{\left|A_{n, j}\right|}\right) \in_{\left|A_{n, j}\right|}(m)$, let $(\pi)$ denote the integer $\frac{m !}{\prod \pi_{t} !}$.

Lemma 3.9 Let $m_{j}=\sum_{\alpha=0}^{\ell_{j}} m_{j, \alpha} p^{\alpha}$. Then

$$
d_{n j}^{m_{j}}=\sum_{\substack{0 \leq \alpha \leq \ell_{j} \\ \pi^{(j, \alpha)} \in A_{n, j} \mid}} \prod_{\alpha=0}^{\ell_{j}}\left(\pi^{(j, \alpha)}\right) h^{\sum^{\alpha} \pi_{i}^{(j, \alpha)}} \sum_{C_{j, i} \in A_{n, j}} p^{\alpha} \mathbf{1} \cdot C_{j, i}
$$

Proof First, we show the formulae above for $m_{j, \alpha}$ and then we extend by direct multiplication.

Proposition 3.10 Let $m=\left(m_{0}, \ldots, m_{n-1}\right)$ be a sequence of non-negative integers, then

$$
d^{m}=\sum_{\substack{0 \leq j \leq n-1,0 \leq \alpha \leq \ell_{j} \\ \pi^{(j, \alpha)} \in \in_{\left|A_{n, j}\right|}\left(m_{j, \alpha}\right)}} \prod_{j=0}^{n-1} \prod_{\alpha=0}^{\ell_{j}}\left(\pi^{(j, \alpha)}\right) h^{\sum_{j} \sum_{\alpha} \pi_{i}^{(j, \alpha)} \sum_{\substack{C_{j, i} \in A_{n, j} \\ 0 \leq j \leq n-1}} p^{\alpha} \mathbf{1} \cdot C_{j, i}}
$$


The following lemma which is of great importance for dealing with Adem relations involving Bockstein operations is proved using appropriate matrices.

Lemma 3.11 Each term of $d_{k+t, s}$ is also a term of $d_{k, s} d_{k+t, k}$. Here $0 \leq s<k$ and $1 \leq t$. Moreover, no term of $d_{k, s} d_{k+t, k}-d_{k+t, s}$ is divisible by $\prod_{k+1}^{k+t} h_{i}$.

In order to prove the main theorem in the next section, the following formula for decomposing Dickson generators will be needed. This formula is a special case of the lemma above. Formulas of this kind might be of interest for other circumstances involving the Dickson algebra. One of them may be the transfer between the Dickson algebra and the ring of invariants of parabolic subgroups.

Lemma 3.12 Let $0 \leq s<k$. Then $d_{k, s} d_{k+1, k}-d_{k+1, s}=$ $\sum_{t=0}^{s-1} d_{k-t-1, s-t}^{p^{t}} d_{k-t-1, k-t-2}^{p^{t+2}} h_{k-t}^{p^{t}}+d_{k-s, 0}^{p^{s}} d_{k-s, k-s-1}^{p^{s+1}}+d_{k-s, 1}^{p^{s-1}} h_{k-s+1}^{p^{s-1}}$.

Proof We shall use induction and the well known formula $d_{k, s}=d_{k-1, s-1}^{p}+$ $d_{k-1, s} h_{k}$.

Lemma 3.13 Each term of $d_{k+q, k} d_{k+t, s}$ is also a term of $d_{k+q, s} d_{k+t, k}$. Here $0 \leq s<k$ and $0 \leq q<t$. Moreover, no term of $d_{k+q, s} d_{k+t, k}-d_{k+q, k} d_{k+t, s}$ is divisible by $\prod_{k+q+1}^{k+t} h_{i}$.

Proof We consider $(k+t) \times(k+t)$ matrices of the following form:

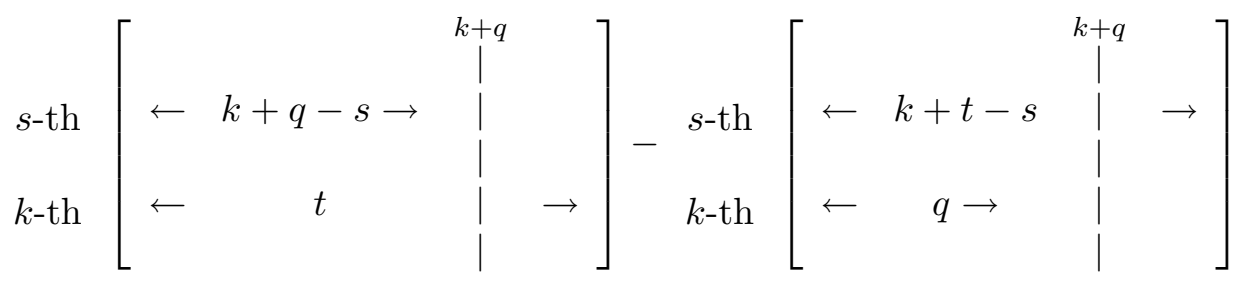

The last column of the matrices above is of size $t-q$. If this column is full of non-zero elements in the last matrix, we require the same in the $k$-th row of the first matrix. Then our matrices under consideration become:

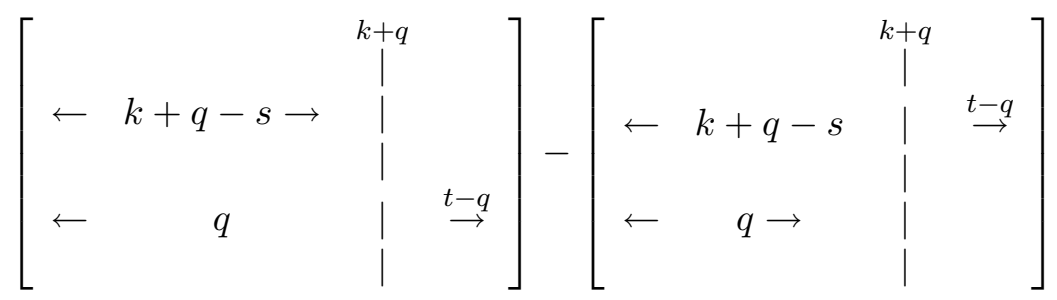


Now the assertion follows because there is no other choice for the first matrix of this kind. For the general case, let the non-zero elements in the last column of the second matrix be $l<t-q$. Then the situation is as follows:



Hence we have to consider the following $(k+q) x(k+q)$ matrices:

$$
\left[\begin{array}{ccc}
\leftarrow & k+q-s & \rightarrow \\
\leftarrow & t-l & \rightarrow
\end{array}\right]-\left[\begin{array}{ccc}
\leftarrow & k+t-s-l & \rightarrow \\
\leftarrow & q & \rightarrow
\end{array}\right]
$$

Here the $s$-th column of the second matrix and the $k$-th column of the first one have been raised to the power $p^{t-q-l}$. Because the exponents are of the right form the assertion follows.

For the rest of this section we recall the ring of invariants $\left(E\left(x_{1}, \ldots, x_{n}\right) \otimes\right.$ $\left.P\left[y_{1}, \ldots, y_{n}\right]\right)^{G L_{n}}$ from [7]. Here $p>2$.

Theorem 3.14 [7] 1) The algebra $(E(n) \otimes S[n])^{B_{n}}$ is a tensor product between the polynomial algebra $B[n]$ and the $\mathbb{Z} / p \mathbb{Z}$-module spanned by the set of elements consisting of the following monomials:

$$
M_{s ; s_{1}, \ldots, s_{m}} L_{s}^{p-2} ; 1 \leq m \leq n, m \leq s \leq n, \text { and } 0 \leq s_{1}<\cdots<s_{m}=s-1 .
$$

Its algebra structure is determined by the following relations:

a) $\left(M_{s ; s_{1}} L_{s}^{p-2}\right)^{2}=0$, for $1 \leq s \leq n, 0 \leq s_{1} \leq s-1$.

b) $M_{s ; s_{1}, \ldots, s_{m}} L_{s}^{p-2}\left(L_{s}^{p-1}\right)^{m-1}=$ $(-1)^{m(m-1) / 2} \prod_{q=1}^{m}\left(\sum_{r=s_{q}+1}^{s} M_{r ; r-1} L_{r}^{p-2} h_{r+1} \ldots h_{s} d_{r-1, s_{q}}\right)$

Here $1 \leq m \leq n, m \leq s \leq n$, and $0 \leq s_{1}<\cdots<s_{m}=s-1$.

2) The algebra $(E(n) \otimes S[n])^{G L_{n}}$ is a tensor product between the polynomial algebra $D[n]$ and the $\mathbb{Z} / p \mathbb{Z}$-module spanned by the set of elements consisting of the following monomials:

$$
M_{n ; s_{1}, \ldots, s_{m}} L_{n}^{p-2} ; 1 \leq m \leq n, \text { and } 0 \leq s_{1}<\cdots<s_{m} \leq n-1 .
$$


Its algebra structure is determined by the following relations:

a) $\left(M_{n ; s_{1}, \ldots, s_{m}} L_{n}^{p-2}\right)^{2}=0$ for $1 \leq m \leq n$, and $0 \leq s_{1}<\cdots<s_{m} \leq n-1$.

b) $M_{n ; s_{1}, \ldots, s_{m}} L_{n}^{(p-2)} d_{n, n-1}^{m-1}=(-1)^{m(m-1) / 2} M_{n ; s_{1}} L_{n}^{p-2} \ldots M_{n ; s_{m}} L_{n}^{p-2}$.

Here $1 \leq m \leq n$, and $0 \leq s_{1}<\cdots<s_{m} \leq n-1$.

The elements $M_{n ; s_{1}, \ldots, s_{m}}$ above have been defined by Mui in [7]. Their degrees are $\left|M_{n ; s_{1}, \ldots, s_{m}}\right|=m+2\left(\left(1+\cdots+p^{n-1}\right)-\left(p^{s_{1}}+\cdots+p^{s_{m}}\right)\right)$ and $\left|L_{n}^{p-2}\right|=$ $2(p-2)\left(1+\cdots+p^{n-1}\right)$.

Definition 3.15 Let $S(E(n))^{B_{n}}$ be the subspace of $(E(n) \otimes S[n])^{B_{n}}$ generated by:

i) $M_{s ; s-1}\left(L_{s}\right)^{p-2}$ for $1 \leq s \leq n$

ii) $\prod_{t=1}^{\ell}\left(M_{s_{2 t-1}+1 ; s_{2 t-1}}\left(L_{s_{2 t-1}+1}\right)^{p-2} M_{s_{2 t}+1 ; s_{2 t}}\left(L_{s_{2 t}+1}\right)^{p-2}\right) / d_{s_{2 t-1}+1,0}$

for $0 \leq s_{1}<\ldots<s_{2 \ell} \leq n-1$,

iii) $M_{s_{1}+1 ; s_{1}}\left(L_{s}\right)^{p-2}$

$$
\prod_{t=1}^{\ell}\left(M_{s_{2 t}+1 ; s_{2 t}}\left(L_{s_{2 t}+1}\right)^{p-2} M_{s_{2 t+1}+1 ; s_{2 t+1}}\left(L_{s_{2 t+1}+1}\right)^{p-2}\right) / d_{s_{2 t}+1,0}
$$

for $0 \leq s_{1}<\ldots<s_{2 \ell+1} \leq n$;

and $S(E(n))^{G L_{n}}$ be the subspace of $(E(n) \otimes S[n])^{G L_{n}}$ generated by:

$M_{n ; s}\left(L_{n}\right)^{p-2}$ for $0 \leq s \leq n-1$,

$\prod_{t=1}^{\ell} M_{n ; s_{2 t-1}, s_{2 t}}\left(L_{n}\right)^{p-2}$ for $0 \leq s_{1}<\ldots<s_{2 \ell} \leq n-1$,

$M_{n ; s_{1}-1}\left(L_{n}\right)^{p-2} \prod_{t=1}^{\ell} M_{n ; s_{2 t}, s_{2 t+1}}\left(L_{n}\right)^{p-2}$ for $0 \leq s_{1}<\ldots<s_{2 \ell+1}<n$.

The following lemmata provide the decomposition of $M_{n ; s, m}\left(L_{n}\right)^{p-2}$ in $S(E(n))^{B_{n}} \otimes B[n]$ and relations between them.

Lemma 3.16 Let $s<\ell$, then $M_{s ; s-1} L_{s}^{p-2} M_{\ell ; \ell-1} L_{\ell}^{p-2}$ can be written with respect to basis elements of $B[k] \otimes S\left(E_{k}\right)^{B_{k}}$.

Lemma 3.17 Let $m<s-1$, then $M_{s ; \ell, s-1} L_{s}^{p-2} M_{m ; m-1} L_{m}^{p-2}$ can be written with respect to basis elements of $S(E(k))^{B_{k}} \otimes B[k]$.

Lemma $3.18 M_{n ; s, m}\left(L_{n}\right)^{p-2}=$

$\sum_{\substack{s \leq q<t \\ m \leq t \leq n-1}} M_{q+1 ; q}\left(L_{q+1}\right)^{p-2} M_{t+1 ; t}\left(L_{t+1}\right)^{p-2} h_{t+2} \ldots h_{n}\left(d_{q, s} d_{t, m}-d_{q, m} d_{t, s}\right) / d_{q+1,0}$.

Here $d_{i, i}=1$ and $d_{i, j}=0$ if $i<j$. 
Corollary 3.19 Let $\kappa=\left[\frac{n+1}{2}\right]$ and $\varepsilon=\left(\epsilon_{1}, \ldots, \epsilon_{n}\right) \in(Z / 2 Z)^{n}$, then $S(E(n))^{G L_{n}}$ is spanned by at most $\kappa$ monomials:

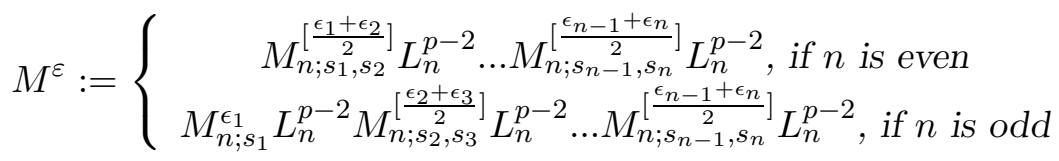

The analogue corollary holds for $S(E(n))^{B_{n}}$.

The Steenrod algebra acts naturally on $S(E(n))^{G L_{n}} \otimes D[n]$ and $S(E(n))^{B_{n}} \otimes$ $B[n]$.

Let $\hat{\imath}: S(E(n))^{G L_{n}} \otimes D[n] \hookrightarrow S(E(n))^{B_{n}} \otimes B[n]$ be the inclusion, then $\hat{\imath}\left(d^{m} M^{\varepsilon}\right)$ means the decomposition of $d^{m} M^{\varepsilon}$ in $S(E(n))^{B_{n}} \otimes B[n]$.

Lemma 3.20 Let $0 \leq s_{1}\left(s_{1}^{\prime}\right)<k_{1}\left(k_{1}^{\prime}\right)<\ldots<s_{l^{\prime}}\left(s_{l^{\prime}}^{\prime}\right)<k_{l^{\prime}}\left(k_{l^{\prime}}^{\prime}\right) \leq n-1$. If $\sum_{0}^{n-1} m_{i}\left(p^{n}-p^{i}\right)+\sum_{1}^{l^{\prime}}\left(p^{n}-p^{s_{i}}-p^{k_{i}}\right)=\sum_{0}^{n-1} m_{i}^{\prime}\left(p^{n}-p^{i}\right)+\sum_{1}^{l^{\prime}}\left(p^{n}-p^{s_{i}^{\prime}}-p^{k_{i}^{\prime}}\right)$, then $s_{i}=s_{i}^{\prime}$ and $k_{i}=k_{i}^{\prime}$. Moreover, if in addition $0 \leq k_{0}\left(k_{0}^{\prime}\right)<s_{1}\left(s_{1}^{\prime}\right)$ and $\sum_{0}^{n-1} m_{i}\left(p^{n}-p^{i}\right)+\left(p^{n}-p^{k_{0}}\right)+\sum_{1}^{l^{\prime}}\left(p^{n}-p^{s_{i}}-p^{k_{i}}\right)=\sum_{0}^{n-1} m_{i}^{\prime}\left(p^{n}-p^{i}\right)+\left(p^{n}-p^{k_{0}^{\prime}}\right)+$ $\sum_{1}^{l^{\prime}}\left(p^{n}-p^{s_{i}^{\prime}}-p^{k_{i}^{\prime}}\right)$, then $s_{i}=s_{i}^{\prime}$ and $k_{j}=k_{j}^{\prime}$.

\section{Calculating the hom-duals and Adem relations}

We start this section by recalling the description of $R[n]^{*}$ as an algebra, for $p$ odd please see May [3] Theorem 3.7 page 29. The analogue Theorem for $p=2$ was given by Madsen who expressed the connection between $R[n]^{*}$ and Dickson invariants back in 1975, 9.

For convenience we shall write $I$ instead of $(I, \varepsilon)$.

Let $I_{n, i}=(\underbrace{0, \ldots, 0}_{i}, \underbrace{1, \ldots, 1}_{n-i})$. Here $0 \leq i \leq n-1$ and $n-i$ denotes the number of p-th powers. The degree $\left|Q_{I_{n, i}}\right|=2 p^{i}\left(p^{n-i}-1\right)\left[2^{n}-2^{i}\right]$ and the $\operatorname{exc}\left(Q_{I_{n, i}}\right)=$ 0 , if $i<n$, and 1 if $i=0$.

Let $J_{n ; i}=(\underbrace{\frac{1}{2}, \ldots, \frac{1}{2}}_{i}, \underbrace{1, \ldots, 1}_{n-i}) x(\underbrace{0, \ldots, 0,1}_{i+1}, \underbrace{0, \ldots, 0}_{n-i-1})$. Here $\varepsilon=(\underbrace{0, \ldots, 0}_{i}, 1, \underbrace{0, \ldots, 0}_{n-i-1})$ and $0 \leq i \leq n-1$. The degree $\left|Q_{J_{n ; i}}\right|=2 p^{i}\left(p^{n-i}-1\right)-1$ and the $\operatorname{exc}\left(Q_{J_{n ; i}}\right)=1$. 
Let $K_{n ; s, i}=(\underbrace{0, \ldots, 0}_{s}, \underbrace{\frac{1}{2}, \ldots, \frac{1}{2}}_{i-s}, \underbrace{1, \ldots, 1}_{n-i}) x(\underbrace{0, \ldots, 0}_{s}, \underbrace{1,0, \ldots, 0,1}_{i-s+1}, \underbrace{0, \ldots, 0}_{n-i})$. Here $\varepsilon=$ $(\underbrace{0, \ldots, 0}_{s}, \underbrace{1,0, \ldots, 0,1}_{i-s}, \underbrace{0, \ldots, 0}_{n-i})$ and $0 \leq s<i \leq n-1$. There are two Bockstein operations in this element: at the $s$-th and $i$-th position from the left. The degree $\left|Q_{K_{n ; s, i}}\right|=2\left(p^{i}\left(p^{n-i}-1\right)-p^{s}\right)$ and the $\operatorname{exc}\left(Q_{K_{n ; s, i}}\right)=0$.

Let $O_{n, i}=(0, \ldots, 0,1,0, \ldots, 0)$, where there are $n-i$ zeros. Its degree is $\left|e_{O_{n, i}}\right|=$ $2 p^{i-1}(p-1)\left[2^{i-1}\right]$ and $\operatorname{exc}\left(e_{O_{n, i}}\right)=0$. Here $1 \leq i \leq n$.

Let $J_{n, i ; i-1}=(\underbrace{\frac{1}{2}, \ldots, \frac{1}{2}}_{i}, \underbrace{0, \ldots, 0}_{n-i}) x(\underbrace{0, \ldots, 0,1}_{i}, \underbrace{0, \ldots, 0}_{n-i})$. Here $\varepsilon=(\underbrace{0, \ldots, 0}_{i-1}, 1, \underbrace{0, \ldots, 0}_{n-i})$

and $1 \leq i \leq n$. Its degree $\left|Q_{J_{n, i ; i-1}}\right|=2 p^{i-1}(p-1)-1$ and the $\operatorname{exc}\left(Q_{J_{n, i ; i-1}}\right)=$ 1 .

Let $K_{n, i ; s, i-1}=(\underbrace{0, \ldots, 0}_{s}, \underbrace{\frac{1}{2}, \ldots, \frac{1}{2}}_{i-s}, 1, \underbrace{0, \ldots, 0}_{n-i}) x(\underbrace{0, \ldots, 0}_{s}, \underbrace{1,0, \ldots, 0,1}_{i-s}, \underbrace{0, \ldots, 0}_{n-i})$. Here $\varepsilon=(\underbrace{0, \ldots, 0}_{s}, \underbrace{1,0, \ldots, 0,1}_{i-s}, \underbrace{0, \ldots, 0}_{n-i})$ and $0 \leq s<i-1 \leq n-1$. Its degree $\left|Q_{K_{n, i ; s, i-1}}\right|=2\left(p^{i}-p^{s}-p^{i-1}\right)$ and the $\operatorname{exc}\left(Q_{K_{n, i ; s, i-1}}\right)=0$.

Let $\xi_{n, 0}=\left(\left(Q_{0}\right)^{n}\right)^{*}=\left(\left(Q^{0}\right)^{n}\right)^{*}$;

$\xi_{n, i}=\left(Q_{I_{n, i}}\right)^{*}=\left(Q^{\left(p^{i-1}\left(p^{n-i}-1\right), \ldots,\left(p^{n-i}-1\right), p^{n-i-1}, \ldots, p, 1\right)}\right)^{*}, 0 \leq i \leq n-1$;

$\tau_{n ; i}=\left(Q_{J_{n ; i}}\right)^{*}=\left(Q^{\left(p^{i-1}\left(p^{n-i}-1\right), \ldots,\left(p^{n-i}-1\right), p^{n-i-1}, \ldots, p, 1,\right) x \varepsilon}\right)^{*}, 0 \leq i \leq n-1$;

$\sigma_{n ; s, i}=\left(Q_{K_{n ; s, i}}\right)^{*}=\left(Q^{\left(p^{i-1}\left(p^{n-i}-1\right)-p^{s-1}, \ldots, p^{i-s-1}\left(p^{n-i}-1\right), \ldots, p^{n-i}-1, p^{n-i-1}, \ldots, p, 1\right) x \varepsilon}\right)^{*}$,

$0 \leq s<i \leq n-1$;

$\zeta_{n, i}=\left(e_{O_{n, i}}\right)^{*}=\left(e^{\left(p^{i-2}(p-1), \ldots,(p-1), 1,0, \ldots, 0\right)}\right)^{*}, 1 \leq i \leq n$;

$\nu_{n, i ; i-1}=\left(e_{J_{n, i ; i-1}}\right)^{*}=\left(e^{\left(p^{i-2}(p-1), \ldots,(p-1), 1,0, \ldots, 0\right) x \varepsilon}\right)^{*}, 1 \leq i \leq n$;

$v_{n, i ; s, i-1}=\left(e_{K_{n, i ; s, i-1}}\right)^{*}=$

$\left(e^{\left(p^{i-1}\left(p^{n-i}-1\right)-p^{s-1}, \ldots, p^{i-s-1}\left(p^{n-i}-1\right), \ldots, p^{n-i}-1, p^{n-i-1}, \ldots, p, 1,0, \ldots, 0\right) x \varepsilon}\right)^{*}$,

$0 \leq s<i-1 \leq n-1$.

Theorem 4.1 (Madsen $p=2$, May $p>2$ ) As an $A$ algebra $R[n]^{*} \cong$ free associative commutative algebra generated by $\left\{\xi_{n, i}, \tau_{n ; i}\right.$, and $\sigma_{n ; s, i} \mid 0 \leq i \leq$ $n-1$, and $0 \leq s<i\},\left[\left\{\xi_{n, i} \mid 0 \leq i \leq n-1\right\}\right]$, modulo the following relations:

a) $\tau_{n ; i} \tau_{n ; i}=0$.

b) $\tau_{n ; s} \tau_{n ; i}=\sigma_{n ; s, i} \xi_{n, 0}$. Here $0 \leq s<i \leq n-1$.

c) $\tau_{n ; s} \tau_{n ; i} \tau_{n ; j}=\tau_{n ; s} \sigma_{n ; i, j} \xi_{n, 0}$. Here $0 \leq s<i<j \leq n-1$.

d) $\tau_{n ; s} \tau_{n ; i} \tau_{n ; j} \tau_{n ; k}=\sigma_{n ; s, i} \sigma_{n ; j, k} \xi_{n, 0}^{2}$. Here $0 \leq s<i<j<k \leq n-1$. 
Theorem $4.2[5] \quad R[n]^{*} \approx S(E(n))^{G L_{n}} \otimes D[n]\left[R[n]^{*} \approx D[n]\right]$ and $T[n]^{*} \approx$ $S(E(n))^{B_{n}} \otimes B[n]\left[T[n]^{*} \approx B[n]\right]$ as algebras over the Steenrod algebra and the isomorphism $\Phi$ is given by $\Phi\left(\xi_{n, i}=\left(Q_{I_{n, i}}\right)^{*}\right)=d_{n, n-i}, \Phi\left(\tau_{n ; i}=\left(Q_{J_{n ; i}}\right)^{*}\right)=$ $M_{n ; i}\left(L_{n}\right)^{p-2}, \Phi\left(\sigma_{n ; s, i}=\left(Q_{K_{n ; s, i}}\right)^{*}\right)=M_{n ; s, i}\left(L_{n}\right)^{p-2}$. Here $0 \leq i \leq n-1$ and $0 \leq s<i$.

$\Phi\left(\zeta_{n, i}=\left(e_{O_{n, i}}\right)^{*}\right)=h_{i}, \Phi\left(\nu_{n, i ; i-1}=\left(e_{J_{n, i ; i-1}}\right)^{*}\right)=M_{i ; i-1}\left(L_{i}\right)^{p-2}$, $\Phi\left(v_{n, i ; s, i-1}=\left(e_{K_{n, i ; s, i-1}}\right)^{*}\right)=\left(M_{s+1 ; s}\left(L_{s+1}\right)^{p-2} M_{i ; i-1}\left(L_{i}\right)^{p-2}\right) / d_{s+1,0}$. Here $1 \leq i \leq n$ and $0 \leq s<i-1$.

Under isomorphism $\Phi$ in Theorem 4.2 we identify $R[n]^{*}$ with $S(E(n))^{G L_{n}} \otimes$ $D[n]$ and $B[n]^{*}$ with $S(E(n))^{B_{n}} \otimes B[n]$.

The set $\underset{3}{\mathrm{~T}}[n]$ and $\mathrm{R}[n]$ of admissible monomials in $T[n]$ and $R[n]$ provide vector space bases respectively. Let $\theta: \mathrm{R}[n] \longmapsto \underset{3}{\mathrm{~T}}[n]$ be the map given by

$$
\theta\left(Q_{I}\right)=e_{I}
$$

The image of the dual of these bases are denoted by $\underset{3}{\mathrm{~T}}[n]^{*}$ in $\Phi(T[n])^{*}=$ $S(E(n))^{B_{n}} \otimes B[n]$ and $\mathrm{R}[n]^{*}$ in $\Phi(R[n])^{*}=S(E(n))^{G L_{n}} \otimes D[n]$. Of course there are also the bases of monomials which are denoted by $\beta_{n}\left(S(E(n))^{B_{n}} \otimes B[n]\right)$ and $\beta_{n}\left(S(E(n))^{G L_{n}} \otimes D[n]\right)$ respectively. We shall note that $\underset{3}{\mathrm{~T}}[n]^{*}=\beta_{n}\left(S(E(n))^{B_{n}} \otimes B[n]\right)$.

The decomposition relations between the other two bases are not obvious and this is the first topic of this section. Campbell, Peterson and Selick provided a method to pass from $\beta_{n}(D[n])$ to $\mathrm{R}[n]^{*}$ in 2 . We shall establish some machinery to work with those bases.

Definition 4.3 Let $\chi_{\min }$ and $\chi_{\max }$ be the set functions from $\beta\left(S(E(n))^{G L_{n}} \otimes\right.$ $D[n])\left(B\left(B[n] \otimes S\left(E_{n}\right)^{B_{n}}\right)\right)$ to the monoid $\left\langle\mathbb{N}, \frac{1}{2}\right\rangle^{n} \times(\mathbb{Z} / 2 \mathbb{Z})^{n}$ given by

1) $\chi_{\min }\left(d_{n, i}\right)=I_{n, i}, \chi_{\max }\left(d_{n, i}\right)=\left(p^{n-i}, \ldots, p^{n-i}, 0, \ldots, 0\right) x(0, \ldots, 0)$;

2) $\chi_{\min }\left(M_{n ; s} L_{n}^{(p-2)}\right)=J_{n ; s}$,

$$
\chi_{\max }\left(M_{n ; s} L_{n}^{(p-2)}\right)=(\underbrace{\frac{1}{2}, \ldots, \frac{1}{2}}_{s}, \underbrace{1 \frac{1}{2}, \ldots, 1 \frac{1}{2}}_{n-s-1}, 1) x(0, \ldots, 0,1) \text {; }
$$

3) $\chi_{\min }\left(M_{n ; s, m} L_{n}^{(p-2)}\right)=K_{n ; s, m}$ and

$$
\chi_{\max }\left(M_{n ; s, m} L_{n}^{(p-2)}\right)=(\underbrace{0, \ldots, 0}_{m}, \underbrace{\frac{1}{2}, \ldots, 1 \frac{1}{2}}_{n-m-1}, 1) x(\underbrace{0, \ldots, 0}_{m}, \underbrace{1,0, \ldots, 0,1}_{n-m}) ;
$$

and the rule $\chi_{\min }\left(d d^{\prime} M M^{\prime}\right)=\chi_{\min }(d)+\chi_{\min }\left(d^{\prime}\right)+\chi_{\min }(M)+\chi_{\min }\left(M^{\prime}\right)$. Here $d, d^{\prime} \in ß(D[n])$ and $M, M^{\prime} \in ß\left(S(E(n))^{G L_{n}}\right)$. The same holds for $\chi_{\max }$. 
Note that the function $\chi_{\text {min }}$ always provides an admissible element and $\hat{\imath}\left(d_{n, i}\right)$ contains a monomial with a unique admissible sequence, namely $h^{\chi_{\min }\left(d_{n, i}\right)}$, and a monomial with a unique maximal sequence, namely $h^{\chi_{\max }\left(d_{n, i}\right)}$. The same is true for elements $M_{n ; s-1} L_{n}^{p-2}$ and $M_{n ; s, m} L_{n}^{p-2}$. Moreover, $\hat{\imath}\left(d_{n}^{m} M\right)$ might contain a number of monomials with admissible sequences and this is the main point of investigation because of its applications in [6. Namely, those monomials provide possible candidates for $\left(d_{n}^{m} M\right)^{*}$. Primitives in $R$ are well known and so are their duals as generators in $R^{*}$. But it is not the case for their expression with respect to the Dickson algebra. On the other hand, the action on the Dickson algebra is well known on $S(E(n))^{G L_{n}} \otimes D[n]$ and hence it is easier to compute the annihilator ideal in the mod- $p$ cohomology of a certain finite loop space.

Definition 4.4 Let $\Psi$ be the correspondence between $\beta_{n}\left(S(E(n))^{G L_{n}} \otimes D[n]\right)$ and $\mathrm{R}[n]$ given by $d \longmapsto \Psi(d)=Q_{\chi_{\min }(d)}$ and the corresponding one between $\beta_{n}\left(S(E(n))^{B_{n}} \otimes B[n]\right)$ and T⿱ $[n]$ denoted by $\Psi_{T}$ where



The maps $\Psi$ and $\Psi_{T}$ are set bijections.

Let $\iota$ be the map

$$
\iota: \beta_{n}\left(S(E(n))^{G L_{n}} \otimes D[n]\right) \longmapsto \beta_{n}\left(S(E(n))^{B_{n}} \otimes B[n]\right)
$$

defined by $\iota(d)=h^{\chi_{\min }(d)}$.

Note that $e_{\chi_{\max }(d)}, e_{\chi_{\min }(d)} \in \underset{3}{\mathrm{~T}}[n]$. The following diagram is commutative.

$$
\begin{array}{ccccc}
\beta_{n}\left(S(E(n))^{B_{n}} \otimes B[n]\right) & \stackrel{\iota}{\longleftarrow} & \beta_{n}\left(S(E(n))^{G L_{n}} \otimes D[n]\right) & \stackrel{\chi_{\min }}{\longrightarrow} & \left\langle\mathbb{N}, \frac{1}{2}\right\rangle^{n} \times(\mathbb{Z} / 2 \mathbb{Z})^{n} \\
\downarrow \Psi_{T} & & \downarrow \Psi & \swarrow \\
\mathrm{T}[n] & \stackrel{\theta}{\longleftarrow} & \underset{3}{\mathrm{R}}[n] & &
\end{array}
$$

Definition 4.5 A monomial in $\beta_{n}\left(S(E(n))^{B_{n}} \otimes B[n]\right)$ is called admissible if it is an element of $\iota\left(\beta_{n}\left(S(E(n))^{G L_{n}} \otimes D[n]\right)\right)$.

Lemma 4.6 Let $h^{J} M^{\varepsilon} \in S(E(n))^{B_{n}} \otimes B[n]$. The following are equivalent:

i) $h^{J} M^{\varepsilon}$ is admissible;

ii) $j_{t} \leq j_{t+1}$ for $t=1, \ldots, n-1$ and $h^{J}$ is divisible by $\prod_{t=0}^{l}\left(h_{s_{2 t+1}+2} \ldots h_{n}\right)^{\epsilon_{2 t+1}}$ for 
$\kappa$ odd (see [3.19); or $\prod_{t=1}^{l}\left(h_{s_{2 t}+2} \ldots h_{n}\right)^{\epsilon_{2 t}}$, otherwise. If $s_{2 t+1}+2$ or $s_{2 t+2}+2=$ $n+1$, then the corresponding product must be 1 .

iii) $\rho\left(\Psi_{T}\left(h^{J} M^{\varepsilon}\right)\right)$ is admissible in $R[n]$.

Proof This follows from the following relation:

$M_{k ; s} L_{k}^{p-2}=M_{s+1 ; s} L_{s+1}^{p-2} h_{s+1} \ldots h_{k}+\sum_{t=2}^{k-s} M_{s+t ; s+t-1} L_{s+t}^{p-2} d_{s+t-1, s} h_{s+t+1} \ldots h_{k}$. Explicitly, if $h^{J^{\prime}}=h^{J} / \prod_{t=0}^{l}\left(h_{s_{2 t+1}+2} \ldots h_{n}\right)^{\epsilon_{2 t+1}}$, then $\chi_{\min }\left(d^{m} M^{\varepsilon}\right)=\left(J^{\prime}, \varepsilon\right)$. Here $d^{m} M^{\varepsilon}=\prod_{i=0}^{n-1} d_{n, i}^{m_{i}} M_{n ; s_{1}}^{\epsilon_{1}} L_{n}^{p-2} M_{n ; s_{2}, s_{3}}^{\left[\frac{\epsilon_{2}+\epsilon_{3}}{2}\right]} L_{n}^{p-2} \ldots M_{n ; s_{n-1}, s_{n}}^{\left[\frac{\epsilon_{n-1}+\epsilon_{n}}{2}\right]} L_{n}^{p-2}$ and $m_{t}=j_{t}^{\prime}-j_{t-1}^{\prime}$, $m_{0}=j_{0}^{\prime}$.

Firstly, we shall show that $\rho^{*} \equiv \hat{\imath}, \hat{\imath}$ as in 3, i.e. for any $e_{I} \in T[n]$ and $d^{m} M^{\varepsilon}$,

$$
\left\langle d^{m} M^{\varepsilon}, \rho\left(e_{I}\right)\right\rangle=\left\langle\hat{\imath}\left(d^{m} M^{\varepsilon}\right), e_{I}\right\rangle .
$$

Here, $\langle-,-\rangle$ is the Kronecker product. This is done by studying all monomials in $T[n]$ which map to primitives in $R[n]$ after applying Adem relations.

Let $n(m x \varepsilon)=\sum m_{i}+\kappa$. Let $\psi_{n(m x \varepsilon)}: R[n] \rightarrow \stackrel{n(m x \varepsilon)}{\otimes} R[n]$ be the iterated coproduct $n(m x \varepsilon)$ times. Let $J$ be admissible, $\rho e_{J}=Q_{J}$, then

$$
\begin{aligned}
\psi Q_{J} & =\psi \rho e_{J}=\rho \psi e_{J}=\rho\left(\Sigma \pm e_{J_{1}} \otimes \cdots \otimes e_{J_{n(m x \varepsilon)}}\right), \quad \Sigma J_{i}=J \\
\psi_{n(m x \varepsilon)} Q_{J} & =\Sigma a_{J_{1}, \ldots, J_{n(m x \varepsilon)}} Q_{J_{1}^{\prime}} \otimes \cdots \otimes Q_{J_{n(m x \varepsilon)}^{\prime}} .
\end{aligned}
$$

Since $J_{i}$ may not be in admissible form, after applying Adem relations we have $J_{i}^{\prime} \leq J_{i}$.

$$
\begin{aligned}
& \left\langle d^{m} M^{\varepsilon}, \rho e_{I}\right\rangle=\left\langle\prod_{i=0}^{n-1} d_{n, i}^{m_{i}} M^{\varepsilon}, \psi_{n(m x \varepsilon)} \rho e_{I}\right\rangle=\left\langle\prod_{i=0}^{n-1} d_{n, i}^{m_{i}} M^{\varepsilon}, \rho \psi_{n(m x \varepsilon)} e_{I}\right\rangle= \\
& \left\langle\prod_{i=0}^{n-1} d_{n, i}^{m_{i}} M^{\varepsilon}, \sum_{I_{j}} \bigotimes_{j}^{n(m x \varepsilon)} \rho e_{I_{j}}\right\rangle=\sum_{I_{j}} \prod_{j}^{n(m)}\left\langle d_{n, i}^{j}, \rho e_{I_{j}}\right\rangle \prod_{j}^{n(\varepsilon)}\left\langle M_{n ; s_{j-1}, s_{j}}^{\left[\frac{\epsilon_{j-1}+\epsilon_{j}}{2}\right]} L_{n}^{p-2}, \rho e_{I_{j}}\right\rangle .
\end{aligned}
$$

Lemma 4.7 Let $d^{m}=\prod_{i=1}^{n} d_{n, i}^{m_{i}}$. Then $\iota\left(d^{m}\right)=\prod_{t=1}^{n} h_{t}^{t-1} \sum^{t=0} m_{i}$ and $\left(\iota\left(d^{m}\right)\right)^{*}=e_{m_{0}} e_{m_{0}+m_{1}} \ldots e_{m_{0}+\ldots m_{n-1}}$.

Lemma 4.8 Let $I=\chi_{\max }\left(d_{n, n-i}\right)$, then $\rho\left(e_{I}\right)=Q_{I_{n, n-i}}=\Psi\left(d_{n, n-i}\right)$ in $R[n]$. 
Proof By direct computation.

Lemma 4.9 Let $e_{I} \in T[n]$ be such that $e_{I}=\Phi^{-1}\left(\prod_{s=1}^{i} h_{j_{s}}^{p^{n-i+s-j_{s}}}\right)$ in (1). Here $1 \leq j_{1}<\ldots<j_{i} \leq n$. Then $\rho\left(e_{I}\right)=Q_{n, n-i}=\Psi\left(d_{n, n-i}\right)$ in $R[n]$.

Proof The sequence $I$ is given by:

$$
(\underbrace{0, \cdots, 0, p^{n-i+1-j_{1}}}_{j_{1}}, \underbrace{0, \cdots, 0, p^{n-i+2-j_{2}}}_{j_{i-2}-j_{1}}, \cdots, \underbrace{0, \cdots, 0, p^{n-j_{i}}}_{j_{i}-j_{i-1}}, \underbrace{0, \cdots, 0}_{n-j_{i}})
$$

Please note the analogy between $I$ above and the corresponding row of a matrix in $A_{n, n-i}$ in section 3 . Here $p^{m}:=0$, whenever $m<0$. We shall work out the first steps to describe the idea of the proof. First, we consider the last $n-i+1$ elements of $\chi_{\max }\left(d_{n, n-i}\right):\left(p^{n-i}, 0, \ldots, 0\right)$ which becomes $(\underbrace{0, \cdots, 0, p^{n-j_{i}}}_{j_{i}-j_{i-1}}, \underbrace{0, \cdots, 0}_{n-j_{i}})$. Thus applying Adem relations on certain positions on $Q_{\chi_{\max }\left(d_{n, n-i}\right)}, Q_{I}$ is obtained and the lemma follows.

Proposition 4.10 Let $e_{I} \in T[n]$ be the hom-dual of a monomial $h^{J} \in T[n]$ such that $\left|h^{J}\right|=2\left(p^{n}-p^{n-i}\right)$ and $h^{J}$ is not a summand in (1). Then $\rho\left(e_{I}\right)=0$ in $R[n]$.

Proof Please see:

http: //www.maths.warwick.ac.uk/agt/ftp/aux/agt-4-13/full.ps.gz $\square$

Now the following theorem is easily deduced because $R[n]$ is a coalgebra, the map $\rho$ is a coalgebra map, and primitives which do not involve Bockstein operations have been considered.

Theorem 4.11 Let $\rho^{\prime}$ be the restriction of $\rho$ between the subcoalgebras $T^{\prime}[n]$ and $R^{\prime}[n]$ where no Bockstein operations are allowed. Let $\hat{\imath}^{\prime}: D[n] \hookrightarrow B[n]$ be the natural inclusion. Then $\left(\rho^{\prime}\right)^{*} \equiv \hat{\imath}^{\prime}$, i.e. for any $e_{I} \in T[n]$ and $d^{m}=$ $\prod_{i=0}^{n-1} d_{n, i}^{m_{i}} \in D[n]$

$$
\left\langle d^{m}, \rho^{\prime}\left(e_{I}\right)\right\rangle=\left\langle\hat{\imath}^{\prime}\left(d^{m}\right), e_{I}\right\rangle .
$$

We shall extend last Theorem to cases including Bockstein operations as well.

Please recall that $\chi_{\min }\left(M_{n ; s} L_{n}^{(p-2)}\right)=J_{n ; s}$. 
Proposition 4.12 a) Let $J=J_{n, t ; t-1}+\left(I_{t-1, s}^{\prime} \oplus 0_{n-t+1}\right)+I_{n, t}$ such that $\rho^{\prime}\left(e_{I_{t-1, s}^{\prime}}\right)=Q_{I_{t-1, s}}$ for $s+1 \leq t \leq n$. Then $\rho\left(e_{J}\right)=Q_{J_{n ; s}}=\Psi\left(M_{n ; s} L_{n}^{p-2}\right)$.

b) Let $J$ be a sequence of length $n$ such that $|J|=2\left(p^{n}-p^{s}\right)-1$ and $J$ is not of the form described in a), then $\rho\left(e_{J}\right)=0$.

c) $\hat{\imath}\left(M_{n ; s} L_{n}^{p-2}\right)=\rho^{*}\left(M_{n ; s} L_{n}^{p-2}\right)$.

Proof Please see: http://www.maths.warwick.ac.uk/agt/ftp/aux/agt-4-13/full.ps.gz

Lemma 4.13 a) Let the sequences $K_{n, t+1 ; q, t}$ and $I_{n, t+1}$, then $\rho e_{\left(K_{n, t+1 ; q, t}+I_{n, t+1}\right)}=Q_{K_{n ; q, t}}$.

b) Let the sequence $K=K_{n ; q, t}+\left(I_{q}^{\prime \prime} \oplus 0_{n-q}\right)+\left(I_{t}^{\prime} \oplus 0_{n-t}\right)$ such that $I_{t}^{\prime}=I_{q}^{\prime} \oplus$ $I_{t-q}^{\prime}, \rho\left(e_{I_{q}^{\prime \prime}}\right)=Q_{I^{\prime \prime, s}}$ and $\rho\left(e_{I_{t}^{\prime}}\right)=Q_{I_{t, m}}$. If we allow Adem relations everywhere in the first $t$ positions except at positions between $q$ and $q+1$ from left, then $\rho^{\prime}\left(e_{K}\right)=e_{K^{\prime}}$ where $K^{\prime}=K_{n ; q, t}+\left(I_{q, s}^{\prime \prime} \oplus 0_{n-q}\right)+p^{t-q-m_{2}}\left(I_{q, m_{1}}^{\prime} \oplus 0_{n-q}\right)+\left(0_{q} \oplus\right.$ $\left.I_{t-q, m_{2}}^{\prime} \oplus 0_{n-t}\right)$ or $K^{\prime}=K_{n ; q, t}+p^{t-q-m_{2}}\left(I_{q, s+m_{1}-q}^{\prime} \oplus 0_{n-q}\right)+\left(0_{q} \oplus I_{t-q, m_{2}}^{\prime} \oplus 0_{n-t}\right)$. For the first case $\rho\left(e_{I_{t}^{\prime}}\right)=Q_{I_{t-q, m_{2}}^{\prime}}, \rho\left(e_{I_{q}^{\prime}}\right)=Q_{p^{t-q-m_{2}} I_{q, m_{1}}^{\prime}}$ and $m=m_{1}+m_{2}$, and for the second $s+m_{1} \geq q$ and $\rho\left(e_{I^{\prime \prime}}{ }_{q}+I_{q}^{\prime}\right)=Q_{p^{t-q-m_{2}} I_{q, s+m_{1}-q}^{\prime}}$.

Proof This is an application of theorem 4.11.

Proposition 4.14 a) Let $K=K_{n, t+1 ; s, t}+\left(I_{t}^{\prime} \oplus 0_{n-t}\right)+I_{n, t+1}$ such that $\rho^{\prime}\left(e_{I_{t}^{\prime}}\right)=Q_{I_{t, m}}$ for $m \leq t \leq n-1$. Then $\rho\left(e_{K}\right)=Q_{K_{n ; s, m}}=\Psi\left(M_{n ; s, m} L_{n}^{p-2}\right)$.

b) Let $K=K_{n, m+1 ; t, m}+\left(I_{t}^{\prime} \oplus 0_{n-t}\right)+I_{n, m+1}$ such that $\rho^{\prime}\left(e_{I_{t}^{\prime}}\right)=Q_{I_{t, s}}$ for $s \leq t \leq m-1$. Then $\rho\left(e_{K}\right)=Q_{K_{n ; s, m}}=\Psi\left(M_{n ; s, m} L_{n}^{p-2}\right)$.

c) Let $K=K_{n, t+1 ; q, t}+I+I_{n, t+1}$ for $m \leq q<t \leq n-1$ with $I=I^{\prime}+I^{\prime \prime}$, $I^{\prime}=\left(I_{q}^{\prime} \oplus 0_{n-q}\right), I^{\prime \prime}=\left(I_{t}^{\prime \prime} \oplus 0_{n-t}\right)$ such that: $\rho^{\prime}\left(e_{I_{t}^{\prime \prime}}\right)=Q_{I_{t, m}}$ and $\rho^{\prime}\left(e_{I_{q}^{\prime}}\right)=Q_{I_{q, s}}$ and not of the form $\rho^{\prime}\left(e_{I_{t}^{\prime \prime}}\right)=Q_{I_{t, s}}$ and $\rho^{\prime}\left(e_{I_{q}^{\prime}}\right)=Q_{I_{q, m}}$. Then $\rho\left(e_{K}\right)=$ $Q_{K_{n ; s, m}}=\Psi\left(M_{n ; s, m} L_{n}^{p-2}\right)$.

d) Let $K$ be a sequence of length $n$ such that $|K|=2\left(p^{n}-p^{s}-p^{m}\right)$ and $K$ is not of the form described in a), b) and c) above, then $\rho\left(e_{K}\right)=0$.

e) $\hat{\imath}\left(M_{n ; s, m} L_{n}^{p-2}\right)=\rho^{*}\left(M_{n ; s, m} L_{n}^{p-2}\right)$.

Proof Please see: http://www.maths.warwick.ac.uk/agt/ftp/aux/agt-4-13/full.ps.gz 
Theorem 4.15 Let $\rho: T[n] \rightarrow R[n]$ be the map which imposes Adem relations. Let $\hat{\imath}: S(E(n))^{G L_{n}} \otimes D[n] \hookrightarrow S(E(n))^{B_{n}} \otimes B[n]$ be the natural inclusion. Then $\rho^{*} \equiv \hat{\imath}$, i.e. for any $e_{I} \in T[n]$ and $d^{m} M^{\varepsilon} \in S(E(n))^{G L_{n}} \otimes D[n]$,

$$
\left\langle d^{m} M^{\varepsilon}, \rho\left(e_{I}\right)\right\rangle=\left\langle\hat{\imath}\left(d^{m} M^{\varepsilon}\right), e_{I}\right\rangle
$$

Theorem 4.16 Let $d^{m} M^{\varepsilon}$ be an element of $\beta_{n}\left(S(E(n))^{G L_{n}} \otimes D[n]\right)$, then the following algorithm calculates its image in $R[n]^{*}$ :

$$
d^{m} M^{\varepsilon}=\sum_{J \geq \chi_{\min }\left(d^{m}\right)}\left\langle d^{m}, Q_{J}\right\rangle\left(Q_{\left(J+\chi_{\min }\left(M^{\varepsilon}\right)\right)}\right)^{*}
$$

1) Find all elements $Q_{J}$ in $R[n]$ such that $\left|d^{m}\right|=\left|Q_{J}\right|$ and $J>\chi_{\min }\left(d^{m}\right)$, i.e. solve the Diophantine equation $\sum_{0}^{n-1} k_{i}\left(p^{n}-p^{i}\right)=\left|d^{m}\right|$ for $\left(k_{0}, \ldots, k_{n-1}\right)>$ $\left(m_{0}, \ldots, m_{n-1}\right)$. For each such a sequence $J$, let $J(1)=J-m_{0}(1, \ldots, 1)$ and consider $\Psi^{-1}\left(Q_{J(1)}\right)=d^{J^{\prime}(1)}$ in $D[n]$.

2) Let $d^{m} M^{\varepsilon}=\left(Q_{\chi_{\min }\left(d^{m} M^{\varepsilon}\right)}\right)^{*}$.

3) Let $d^{m(1)}=\frac{\mathrm{d}^{\mathrm{m}}}{\mathrm{d}_{\mathrm{n}, 0}^{\mathrm{m} 0}}$ and $d^{K}$ be an element in step 1) corresponding to the biggest sequence among those which have not been considered yet. If $d^{K(1)}=d^{m(1)}$, then $\alpha_{(K)}=\left\langle d^{m}, Q_{K}\right\rangle=1$. Otherwise, proceed as follows: find the coefficient, $\alpha_{(K)}$, of $\iota\left(d^{K(1)}\right)$ in $\hat{\imath}\left(d^{m(1)}\right), \alpha_{(K)}=\left\langle d^{m}, Q_{K}\right\rangle$. Then add $\alpha_{(K)}\left(Q_{K+\chi_{\min }\left(M^{\varepsilon}\right)}\right)^{*}$ in $d^{m} M^{\varepsilon}$.

\section{4) Repeat step 3).}

Proof Since $R[n]^{*}$ is a free module over $D[n]$ with basis all elements which involve Bockstein operations, the computation of $d^{m} M^{\varepsilon}$ reduces to that of $d^{m}$, i.e.

$$
\begin{aligned}
d^{m} & =\sum_{J \geq \chi_{\min }\left(d^{m}\right)}\left\langle d^{m}, Q_{J}\right\rangle\left(Q_{(J)}\right)^{*} \Rightarrow \\
d^{m} M^{\varepsilon} & =\sum_{J \geq \chi_{\min }\left(d^{m}\right)}\left\langle d^{m}, Q_{J}\right\rangle\left(Q_{\left(J+\chi_{\min }\left(M^{\varepsilon}\right)\right)}\right)^{*}
\end{aligned}
$$

Let $d^{m}=\sum \alpha_{(I)}\left(Q_{I}\right)^{*}$ and $n(m)=\sum_{t=0}^{n-1} m_{t}$. Because of the definition of the hom-dual, we have : $\left\langle d^{m}, Q_{\chi_{\min }\left(d^{m}\right)}\right\rangle=1$ and $\left\langle d^{m}, Q_{I}\right\rangle=a_{(I)} \neq 0$ for a sequence $I$ such that in the $n(m)$-times iterated coproduct:

$$
\psi Q^{I}=\sum_{\Sigma J_{t}=I} e_{J_{1}} \otimes \ldots \otimes e_{J_{n(m)}} \stackrel{\text { Adem }}{=} \sum a_{I_{1}, \ldots, I_{n(m)}} Q_{I_{1}} \otimes \ldots \otimes Q_{I_{n(m)}}
$$


$a_{(I)} \bigotimes_{t=0}^{n-1} \bigotimes_{1}^{m_{t}}\left(Q_{I_{n, t}}\right)$ is a summand. Thus $I \geq \chi_{\min }\left(d^{m}\right)$. Let $I_{1}>\cdots>I_{l}>$ $\chi_{\min }\left(d^{m}\right)$ be all sequences such that $\left|Q_{I_{t}}\right|=\left|Q_{\chi_{\min }\left(d^{m}\right)}\right|$.

We quote from May page 20: if for each $d^{m} M^{\varepsilon}$ we associate its coefficients $a_{(I)}$ as a matrix $\left(a_{\chi_{\min }\left(d^{m} M^{\varepsilon}\right),(I)}\right)$, then this matrix is upper triangular with ones along the main diagonal. This allows us to express one basis element $d^{m} M^{\varepsilon}$ with respect to the dual basis of admissible monomials.

We consider the first sequence $I_{1}$. Our task is to evaluate $\alpha_{\left(I_{1}\right)}$. Let $\psi Q_{I_{1}}$ be the iterated coproduct applied $n(m)$-times. We shall write $I_{1}$ as a sum of $n(m)$ sequences such that each of them is a primitive element of $R[n]$ equals to one of those involved in $\chi_{\min }\left(d^{m}\right)$. This is possible, since $n(m) \geq n\left(\chi_{\min }\left(\Psi^{-1}\left(Q_{I_{1}}\right)\right)\right)$. The common element $d_{n, 0}^{m_{0}}$ between $\Psi^{-1}\left(Q_{\left(I_{1}\right)}\right)$ and $d^{m}$ does not change the coefficient $\alpha_{\left(I_{1}\right)}$, because no Adem relation can reduce $Q_{I_{n, 0}}$ to a smaller sequence. Instead, we consider $Q_{I_{1}-m_{0} I_{n, 0}}\left(d^{J_{1}}=\Psi^{-1}\left(Q_{\left(I_{1}\right)}\right) / d_{n, 0}^{m_{0}}\right)$ and $Q_{\left(\chi_{\min }\left(d^{m}\right)-m_{0} I_{n, 0}\right)}$ $\left(d^{m(1)}=d^{m} / d_{n, 0}^{m_{0}}\right)$. Now the iterated coproduct is applied $n(m(1))$-times.

For the second part of step 3), we use $\psi \rho=\rho \psi$, lemma 4.9 and proposition 4.10. All elements $e_{I} \in T[n]$, which have the property $\rho e_{I}=Q_{I_{n, n-i}}$, are known. Moreover, the dual of those elements, $\left(e_{I}\right)^{*} \in B[n]$, are summands in $\hat{\imath}\left(d_{n, n-i}\right)$. Using commutativity in $D[n]$ induced by symmetry in coproduct, we deduce that the required coefficient is the coefficient of $\iota\left(d^{J_{1}}\right)$ in $\hat{\imath}\left(d^{m(1)}\right)$.

Remark Suppose that $\left(Q_{I}\right)^{*}$ is to be expressed with respect to $\beta_{n}\left(S(E(n))^{G L_{n}} \otimes D[n]\right)$, then one starts with the biggest sequence, say $K(1), \quad \Psi^{-1}\left(Q_{K(1)}\right)=\left(Q_{K(1)}\right)^{*}$, then substitutes in the next element $\Psi^{-1}\left(Q_{K(2)}\right)=\left(Q_{K(2)}\right)^{*}+a_{K(2), K(1)}\left(Q_{K(1)}\right)^{*} \Rightarrow\left(Q_{K(2)}\right)^{*}=\Psi^{-1}\left(Q_{K(2)}\right)-$ $a_{K(2), K(1)} \Psi^{-1}\left(Q_{K(2)}\right)$ and so on.

Let us make some comments. If the degree $m$ of a monomial $d^{m}$ is quite high, then there exist many elements of the same degree such that the dual of their images under $\Phi$ do not appear in $d^{m}$ for a variety of reasons. We shall give a refinement of the algorithm described above through the next lemmas.

Definition 4.17 Let $d^{m}=\prod_{i=0}^{n-1} d_{n, i}^{m_{i}}$ be a monomial in the Dickson algebra and $m_{i}=\sum_{t=0}^{\ell_{i}} a_{i, t} p^{t}$. Let $i_{0}=\max \left\{i \mid m_{i} \neq 0\right\}$ and $0 \leq t<i_{0}$. Let $\delta(t)$ be a positive integer such that $t \leq \gamma(s) \leq n-1$ for $s=1, \ldots, \delta(t)$ and $\sum_{1}^{\gamma(\delta(t))}(n-\gamma(s))=n-t$. 
Let also $\ell_{(t, \gamma(1), \ldots, \gamma(\delta(t)))}=\max \left\{\gamma(s)-\left(\sum_{1}^{s} \gamma(j)\right)+(s-1) n \mid s=1, \ldots, \gamma(\delta(t))\right\}$ and $0 \leq c \leq \min \left\{\ell_{\gamma(s)}-\ell_{(t, \gamma(1), \ldots, \gamma(\delta(t)))}+\sum_{j=1}^{s-1}(n-\gamma(j))\right\}$. We define

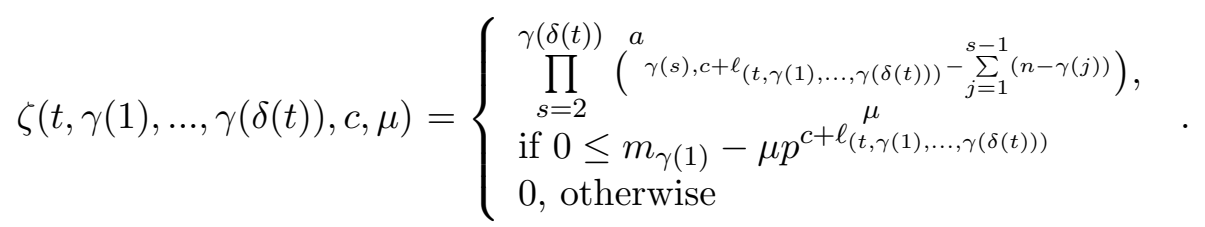

Here $1 \leq \mu \leq \min \left\{a_{\gamma(s), c+\ell_{(t, \gamma(1), \ldots, \gamma(\delta(t)))}-\sum_{j=1}^{s-1}(n-\gamma(j))} \mid s=2, \ldots, \delta(t)\right\}$

Proposition 4.18 Let $d^{m}=\prod_{i=0}^{n-1} d_{n, i}^{m_{i}}$ be a monomial in the Dickson algebra as above. Then $d^{m}$ contains

$$
\left(\Psi\left(d^{m} d_{n, t}^{p^{\ell}(t, \gamma(1), \ldots, \gamma(\delta(t)))} / \prod_{1}^{\gamma(\delta(t))} d_{n, \gamma(s)}^{\left.\ell^{(t, \gamma(1), \ldots, \gamma(\delta(t)))}\right)^{-\sum_{j=1}^{s-1}(n-\gamma(j))}}\right)\right)^{*}
$$

with coefficient

$$
\begin{aligned}
& \sum_{\gamma(1), \ldots, \gamma(\delta(t))} \zeta(t, \gamma(1), \ldots, \gamma(\delta(t)), 0,1)+ \\
& \sum_{\gamma^{\prime}(1), \ldots, \gamma^{\prime}\left(\delta^{\prime}(t)\right)} \prod_{i \in I_{\left(t, \gamma^{\prime}(1), \ldots, \gamma^{\prime}\left(\delta^{\prime}(t)\right)\right)}}\left(\begin{array}{c}
m_{i} \\
\sigma_{i}\left(t, \gamma^{\prime}(1), \ldots, \gamma^{\prime}\left(\delta^{\prime}(t)\right)\right)
\end{array}\right)
\end{aligned}
$$

such that $\ell_{(t, \gamma(1), \ldots, \gamma(\delta(t)))}=\ell_{\left(t, \gamma^{\prime}(1), \ldots, \gamma^{\prime}\left(\delta^{\prime}(t)\right)\right)}^{\prime}$ and

$$
\prod_{1}^{\gamma(\delta(t))} d_{n, \gamma(s)}^{\ell_{(t, \gamma(1), \ldots, \gamma(\delta(t)))}-\sum_{j=1}^{s-1}(n-\gamma(j))}=\prod_{i \in I_{\left(t, \gamma^{\prime}(1), \ldots, \gamma^{\prime}\left(\delta^{\prime}(t)\right)\right)}} d_{n, i}^{\sigma_{i}\left(t, \gamma^{\prime}(1), \ldots, \gamma^{\prime}\left(\delta^{\prime}(t)\right)\right)} .
$$

Here $\{\gamma(1), \ldots, \gamma(\delta(t))\}$ and $\left\{\gamma^{\prime}(1), \ldots, \gamma^{\prime}\left(\delta^{\prime}(t)\right)\right\}$ are partitions of $\{t+1, \ldots, n\}$ of consecutive and non-consecutive elements respectively. For the definition of $I_{\left(t, \gamma^{\prime}(1), \ldots, \gamma^{\prime}\left(\delta^{\prime}(t)\right)\right)}$ and $\sigma_{i}\left(t, \gamma^{\prime}(1), \ldots, \gamma^{\prime}\left(\delta^{\prime}(t)\right)\right)$, please see the second case in the proof bellow because they strongly depend on the particular partition.

Proof Please see: http://www.maths.warwick.ac.uk/agt/ftp/aux/agt-4-13/full.ps.gz

Next we consider a lemma in the "opposite" direction of last Proposition. 
Lemma 4.19 Let $k \leq n-i$ and $i<n$, then: $d_{n, n-i}^{\alpha_{k} p^{k}+\alpha_{0}}=\left(\Psi\left(d_{n, n-i}^{\alpha_{k} p^{k}+\alpha_{0}}\right)\right)^{*}+$ $\left(\begin{array}{c}\alpha_{k} \\ \min \left(\alpha_{k}, \alpha_{0}\right)\end{array}\right)\left(\begin{array}{c}\alpha_{0} \\ \min \left(\alpha_{k}, \alpha_{0}\right)\end{array}\right)\left(\Psi\left(d_{n, n-i-k}^{\min \left(\alpha_{k}, \alpha_{0}\right) p^{k}} d_{n, n-i}^{\left(\alpha_{k}-\min \left(\alpha_{k}, \alpha_{0}\right)\right) p^{k}+\left(\alpha_{0}-\min \left(\alpha_{k}, \alpha_{0}\right)\right)} d_{n, n-i+k}^{\min \left(\alpha_{k}, \alpha_{0}\right)}\right)\right)^{*}$

Proof We consider all admissible sequences in $\left(i\left(d_{n, n-i}^{p^{k}}\right)\right)^{\alpha_{k}}\left(i\left(d_{n, n-i}\right)\right)^{\alpha_{0}}$.

Note that $d_{n, n-i}^{\alpha_{k} p^{k}+\ldots+\alpha_{0}}$ can be computed by repeated use of the formulae in the last lemma for all possible choices.

Remark We must admit that if $m(n)>>0$, then there exist many candidates for $m^{\prime}$ and the bookkeeping described above can not be done by hand. We believe that it is harder but safer to consider all possible choices.

Next, the algorithm which calculates Adem relations using modular invariants is demonstrated.

Proposition 4.20 Let $e_{I} \in T[n]$. The following algorithm computes $\rho\left(e_{I}\right)$ in $R[n]$.

i) Let $\Re=\left\{m=\left(m_{0}, \ldots, m_{n-1}\right)\right\}$ be all solutions of $|I|=\sum_{0}^{n-1} m_{i}\left(p^{n}-p^{i}\right)+$ $\sum_{1}^{l^{\prime}}\left(p^{n}-p^{s_{i}}-p^{k_{i}}\right)$. Note that $s_{i}$ and $k_{i}$ are uniquelly defined by lemma[3.20, Let $K$ be the set of all admissible sequences $K$ such that $|K|=|I|$ and $K \leq I$. Moreover, $Q_{K} \in R[n]$ and $Q_{K}=\Psi^{-1}\left(d^{m} M^{\epsilon}\right)$ for $m \in \Re$.

ii) Let $h^{I^{\prime}}=\Psi_{T}^{-1}\left(e_{I}\right)$ and find $b_{I, K}$ the coefficient of $h^{I^{\prime}}$ in $\hat{\imath}\left(d^{m} M^{\epsilon}\right)$ for all elements of $\Re$.

iii) Compute the image of $d^{m} M^{\epsilon}$ in $(R[n])^{*}$.

iv) Use the Kronecker product to evaluate $\rho\left(e_{I}\right)$ :

Start with the first non-zero $b_{I, K_{1}}, \rho\left(e_{I}\right)$ contains $a_{I, K_{1}} Q_{K_{1}}$; i.e. $\left\langle d^{K_{1}^{\prime}}, \rho\left(e_{I}\right)\right\rangle=$ $a_{I, K_{1}}=b_{I, K_{1}}$. Proceed to the next sequence $K_{2}$ and use $b_{I, K_{2}}$ (whether or not is zero) and the image of $d^{K_{2}^{\prime}}$ to compute the coefficient $a_{I, K_{2}}$ of $Q^{K_{2}}$ in $\rho\left(e_{I}\right)$. Repeat last step for all remaining sequences.

We close this work by making some remarks about evaluating $\rho\left(e_{I}\right)$ using matrices introduced in section 4 . Since $\left(e_{I}\right)^{*}=h^{I^{\prime}}$ is an element of $B[n]$, one has to find all sequences $m=\left(m_{0}, \cdots, m_{n-1}\right)$ such that $d^{m}$ contains $\left(e_{I}\right)^{*}$ as a summand. This is equivalent to find all matrices $C$ such that $\left(e_{I}\right)^{*}=\prod_{t=1}^{n} h_{t}^{(\mathbf{1} \cdot C)_{t-1}}$ 
and then group them in different sets such that each set corresponds to an $m$. The coefficient $\alpha^{\prime} m$ of $Q^{\prime m}$ in $\rho\left(e_{I}\right)$ is a function of the order of the set corresponding to $m$. Given $h^{I^{\prime}}$, there is a great number of choices for $C$ depending on $I^{\prime}$ as the interested reader can easily check and this is the reason for the high complexity of Adem relations.

\section{References}

[1] H.E.A. Campbell, Upper Triangular Invariants, Canad. Math. Bull. Vol. 28 (2), 1985, 243-248.

[2] Campbell H.E.A., Peterson F.P. and Selick P.S.: Self-maps of loop spaces. I. Trans. AMS 293 no1 (1986), pp. 1-39.

[3] Cohen F., Lada T., and May J.P.: The homology of iterated loop spaces. LNM 533, (1975).

[4] Dickson L.E.: A fundamental system of invariants of the general modular linear group with a solution of the form problem. Trans. AMS 12 (1911), 75-98.

[5] Kechagias E. Nondas: Extended Dyer-Lashof algebras and modular coinvariants. Manuscripta Math. 84, 1994 pp. 261-290.

[6] Kechagias E. Nondas: An invariant theoretic description of the primitive elements of the mod-p cohomology of a finite loop space which are annihilated by Steenrod operations. From: "Proceedings of a Workshop on Invariant Theory, April 2002, Queen's University". C.R.M.- A.M.S. Proceedings and Lecture Notes Volume 35 (2003), 159-174.

[7] Huyhn Mui: Modular invariant theory and the cohomology algebras of the symmetric groups, J. Fac. Sci. Univ. Tokyo, IA (1975), 319-369.

[8] Huynh Mui: Homology operations derived from modular coinvariants. Math $\mathrm{Z}, \mathbf{1 7 2}, 85-115,(1985)$

[9] Madsen, I.: On the action of Dyer-Lashof algebra in $H_{*}(B)$. Pacific Journal of Mathematics, 60, 235-275, (1975).

Department of Mathematics, University of Ioannina, 45110 Greece

Email: nkechag@cc.uoi.gr

URL: http://www.math.uoi.gr/ nondas_k

Received: 23 October 2003 Revised: 20 January 2004 